\title{
The new genotypic human calicivirus isolated in Seoul
}

\author{
Dong-Pyou Han', Hyean-Woo Lee', \\ Joon Hyung Sohn ${ }^{1}$, Byung-II Yeh', \\ Jong-Whan Choi ${ }^{1}$ and Hyun-Won Kim ${ }^{1,2}$ \\ ${ }^{1}$ Department of Biochemistry and Institute of Basic Medical \\ Research, Yonsei University Wonju College of Medicine, \\ 62 Ilsandong, Wonju, Kangwondo 220-701, Korea \\ ${ }^{2}$ Corresponding author: Tel, 82-371-741-0283; \\ Fax, 82-371-743-0411; E-mail, kimhwbio@unitel.co.kr
}

Accepted 15 December 1999

Abbreviations: FCV, feline calicivirus; HuCV, human calicivirus; LV, Lordsdale virus; MV, Manchester virus; NV, Norwalk virus; RDRP, RNA-dependent RNA polymerase; ORF, open reading frame; RHDV, rabbit hemorrhagic disease virus; SRSV, small round structured virus; SHV, Southampton virus; SMV, Snow mountain virus; SV, Sapporo virus

\begin{abstract}
A new type of human calicivirus (HuCV) showing the classic cup-shaped surface morphology was identified in the stool sample from a child with symptoms of acute gastroenteritis in Seoul, Korea (SK virus). Genomic RNA was extracted directly from the stool sample, and the nucleotide sequence of $3.2 \mathrm{~kb}$ of the $3^{\prime}$ end of SK virus was determined from cDNA. This region spanned sequences from the RNA-dependent RNA polymerase (RDRP) region in the open reading frame 1 (ORF1) to the $3^{\prime}$ poly A tail. The nonstructural and capsid protein coding sequences were fused in a single ORF as observed in Manchester type (Genogroup III). However, ORF2 of Manchester virus was missing in SK virus. In RDRP region, SK virus showed amino acid and nucleotide identities of $74-75 \%$ and $68-69 \%$ respectively, with those of Manchester virus, while showed $34-46 \%$ and $55-$ $60 \%$ identities respectively with those of other human caliciviruses. However, capsid protein of SK virus showed a partial (29-46\%) amino acid identity with those of other caliciviruses including Manchester type. The closest resemblance in amino acid (97$99 \%)$ and nucleotide sequence $(85-86 \%)$ identities were found in RDRP region with Vanderbijlpark and Pretoria isolates recently found in South Africa. These results suggest that SK virus together with Vanderbijlpark and Pretoria isolates belong to a new type different from Manchester virus.
\end{abstract}

Keywords: SK virus, calicivirus, sequencing, RNA polymerase, capsid protein

\section{Introduction}

The family Caliciviridae is composed of a single-stranded, positive-sense RNA viruses whose nonenveloped, spherical virions are $35-40 \mathrm{~nm}$ in diameter and consist of a single major capsid protein (Schaffer, 1980). Members of this group include feline calicivirus (FCV), vesicular exanthema of swine virus (VESV), rabbit hemorrhagic disease virus (RHDV), and human cali-civirus (HuCV).

HuCVs cause sporadic cases and outbreaks of gastroenteritis (Madeley and Cosgrove, 1976). Typical HuCVs have the distinctive Star of David appearance and surface hollows (Appleton, 1987). Unlike other Caliciviridae, the Norwalk virus (NV) isolated from patients in an outbreak of gastroenteritis in Norwalk City (Adler and Zickl, 1969; Kapikian et al., 1972) did not show the characteristic Star of David appearance, and is called small round structured viruses (SRSVs) based on its unique morpholgy. NV has also been classified within the family Caliciviridae based on the genome data (Jiang et al., 1990; Matsui et al., 1991; Jiang et al., 1993). HuCVs have been classified into at least five distinct strains based upon their reactivity by immune electron microscopy or radio-immunoassay using acute and convalescent phase human sera (Cubitt et al., 1987); Norwalk, Hawaii, Snow Mountain, Tanton, and Sapporo type. Especially, Sapporo strains were antigenically distinct and its sequence in RNA-dependent RNA polymerase (RDRP) region was rather closer to animal caliciviruses than other known human caliciviruses (Matson et al., 1995). Recently, Sapporo type virus was isolated in Manchester city, UK and its genomic structure was thoroughly examined.

Human caliciviruses have a single-stranded positive sense RNA genome of about 7700 nucleotides (Meyers et al., 1991; Carter et al., 1997) that possesses two or three open reading frames (ORF). The ORF1 encodes a large polypeptide containing amino acid motifs found in the picornavirus $2 \mathrm{C}$ helicase, $3 \mathrm{C}$ protease and $3 \mathrm{D}$ RDRP regions. The ORF2 encodes a single viral capsid protein of about $58 \mathrm{kDa}$ (Jiang et al., 1992a). Capsid protein sequence is fused to ORF1 in RHDV and Manchester virus. The function of ORF3 is unknown, although it is conserved in both human and animal caliciviruses where it encodes a small basic protein which may function as an RNA-binding protein (Neill et al., 1991).

Based on the sequence comparisons of the RDRP region, calicividridae was classified into three subgeno- 
groups; I: (Norwalk related), II: (Snow Mountain or Hawaii virus related), and III: (Sapporo and Manchester viruses) (Lambden and Clark, 1995). The recent study showed that the genome organization of the genogroup III isolates was rather closely resembled that of RHDV. In this investigation, characterization of genome sequence of the calicivirus isolated from a patient with gastroenteritis in Seoul (SK virus) indicated a distinct genome structure from other known HuCVs.

\section{Materials and Methods}

\section{Identification of virus and isolation of HuCV from stool specimens}

The classic human enteric caliciviruses were identified by electron microscopy and RT-PCR in stool samples obtained from a 10 months old infant with an acute diarrhea. HuCV from $10 \mathrm{~g}$ stool sample was purified by the following procedure (Jiang et al., 1990). A 10\% solution of stool samples in PBS was first washed by low speed centrifugation at 3,000 rpm for $15 \mathrm{~min}$, and the washing step was repeated two times. The supernatant was recentrifuged at $15,000 \mathrm{rpm}$ for $60 \mathrm{~min}$ in a SS34 rotor (Sorvall), the resultant supernatant was concentrated by pelleting at $40,000 \mathrm{rpm}$ for $120 \mathrm{~min}$ in a RP70T rotor (Hitachi, SCP85H). The pellet was fractionated by cesium chloride gradient centrifugation according to Jiang et al. (1990) and each fraction was examined for HuCV by RT-PCR with primers p36/p51.

\section{Electron microscopy of fecal specimens}

A specimen obtained from a child with gastroenteritis was processed for negative contrast electron microscopy (Middleton et al., 1977). Negative-contrast stain EM was performed by mixing a small portion of crude stool with $1 \%$ ammonium acetate and then placing a drop of the suspension on an EM polyvinyl formal carbon-coated grid (Ted Pella Inc.). After a few seconds, excess fluid was removed with filter paper, and a drop of $2 \%$ phosphotungstic acid ( $\mathrm{pH} 7.0)$ was added to the grid. Grids were placed under an ultraviolet light for $2 \mathrm{~min}$ and then observed in the EM (JEM-1200EX II).

\section{Isolation of HuCV RNA}

HuCVs RNA was isolated from fecal specimen or the samples fractioned by $\mathrm{CsCl}$ gradient centrifugation with TRIzol Reagent (Gibco BRL) according to the method of Levett et al. (1996). A 10\% suspension of the stool specimen in PBS was washed by centrifugation at 3,000 $\mathrm{rpm}$ in a microcentrifuge for $30 \mathrm{~min}$. The supernatant was recentrifuged for $15 \mathrm{~min}$ at $10,000 \mathrm{rpm}$ and further washed by centrifugation at $10,000 \mathrm{rpm}$ for additional 15 min. A $100 \mu$ volume of the stool suspension was added to $1.0 \mathrm{ml}$ of TRIzol reagent in a microcentrifuge tube. After incubation at room temperature for $5 \mathrm{~min}$, the preparation was mixed by shaking with $0.2 \mathrm{ml}$ of chloroform, and after centrifugation at $12,000 \mathrm{rpm}$ for $10 \mathrm{~min}$, the aqueous layer was removed and mixed with the same volume of isopropanol at room temperature for $10 \mathrm{~min}$. The pellet obtained after centrifuging at $12,000 \mathrm{rpm}$ for $10 \mathrm{~min}$ at $4^{\circ} \mathrm{C}$ was washed with $75 \%$ ethanol, dried, and resuspended in $20 \mu \mathrm{l}$ of Rnase-free water.

\section{Primers for RT-PCR and CDNA library.}

The primers used for RT-PCR and cDNA library of HuCV genome are listed in Table 1. A primer for cDNA library was designed for HuCV cDNA synthesis using oligo $d(T)$ sequence. A primer set p36/p51 which was used for detection of HuCV in the stool specimen was previously described by Ando et al. (1994). The other primer set, $\mathrm{p} 6 \mathrm{H} / \mathrm{pM} 95$ were referred from the nucleotide sequences of HuCV cDNAs obtained by RT-PCR and from cDNA library.

\section{Construction of cDNA library}

A single stranded cDNA for the construction of cDNA library was synthesized using the cDNA synthesis kit (Amersham Life Science) with the primer T25VN and HuCV RNA for template. After the synthesis of the second strand, the reaction mixture was extracted once with phenol-chloroform and once with chloroform followed by ethanol precipitation. The CDNA was ligated with the EcoRI adaptor, and unreacted adaptor was removed through spin column according to the procedure of CDNA rapid adaptor kit (Amersham Life Science).

To detect the sequences which contain HuCV cDNAs, cDNA library was constructed in pBluescript SK(+) with cohesive end ligation into the EcoRI site. White colonies from transformed E. coli TOP10' cells were picked, and minipreps of plasmid DNA were performed for each clone.

Table 1. Primers for RT-PCR and cDNA library

\begin{tabular}{ccll}
\hline cDNA & Position of cDNA (Length) & \multicolumn{1}{c}{ Names(Polarity) and Sequences (Position) of primers } & Methods \\
\hline a & $1-416(416)$ & p36(+); ATAAAAGTTGGCATGAACA & RT-PCR \\
& & p51(-); GTTGACACAATCTCTCATCATCATC & R6H(+); GCCATTCACTAGTGTTGTCAAC \\
b & $263-1761(1499)$ & pM95(-);GCTCAATAGGCAGTGTTGACCAAC & RT \\
& & $\mathrm{T}_{25} \mathrm{VN}(-) ;(\mathrm{T})_{25}(\mathrm{~A} / \mathrm{G} / \mathrm{C})(\mathrm{A} / \mathrm{G} / \mathrm{C} / \mathrm{T})$ & cDNA library \\
\hline
\end{tabular}


Clones containing insert were identified after electrophoresis of the plasmids DNA in an agarose gel, and plasmids with insert DNA were sequenced by the dideoxychain termination method (Sanger et al., 1977) using the Sequenase version 2.0 (USB). Internal primers were synthesized to complete the sequencing of both strands of each clone.

\section{RT-PCR}

RT-PCR was performed as described previously (Jiang et al., 1992b). $5 \mu \mathrm{l}$ of purified viral RNA was reverse transcribed for $1 \mathrm{~h}$ at $42^{\circ} \mathrm{C}$ with $10 \mathrm{U}$ of avian myeloblastosis virus reverse transcriptase in $25 \mu \mathrm{l}$ of PCR buffer containing $10 \mathrm{mM}$ Tris- $\mathrm{HCl}(\mathrm{pH} 8.3), 1.5 \mathrm{mM} \mathrm{MgCl}_{2}, 50$ $\mathrm{mM} \mathrm{KCl}, 3.3 \mathrm{mM}$ each dATP, dCTP, dGTP, and dTTP, $1.0 \mathrm{M}$ primer, and $40 \mathrm{U}$ of RNasin. PCR was performed by adding $70 \mu \mathrm{l}$ of PCR buffer containing the second primer and $5 \mathrm{U}$ of Ex. Taq DNA polymerase (Takara Biomedicals) to the RT reaction mixture. A negative control in which distilled water was substituted for CDNA was included in all sets of PCRs. DNA was amplified for 35 cycles consisted of denaturation for $1 \mathrm{~min}$ at $94^{\circ} \mathrm{C}$, annealing for $1 \mathrm{~min}$ at $55^{\circ} \mathrm{C}$, and extension for $1 \mathrm{~min} / 500$ bp at $72^{\circ} \mathrm{C}$. The first denaturation time was increased to $3 \mathrm{~min}$, and the final extension time was increased to 3$5 \mathrm{~min}$. The PCR products were analyzed by agarose gel electrophoresis and visualized by staining with ethidium bromide and UV illumination. PCR products were separated on $1.0 \%$ SeaKem agarose (FMC Bio Products, $\mathrm{MD}$ ), and a portion of the gel that corresponded to the position of expected PCR products was cut out. CDNAs was purified by binding to silica ('MERmaid' Bio101) following extraction from agarose gels. Extracted DNA

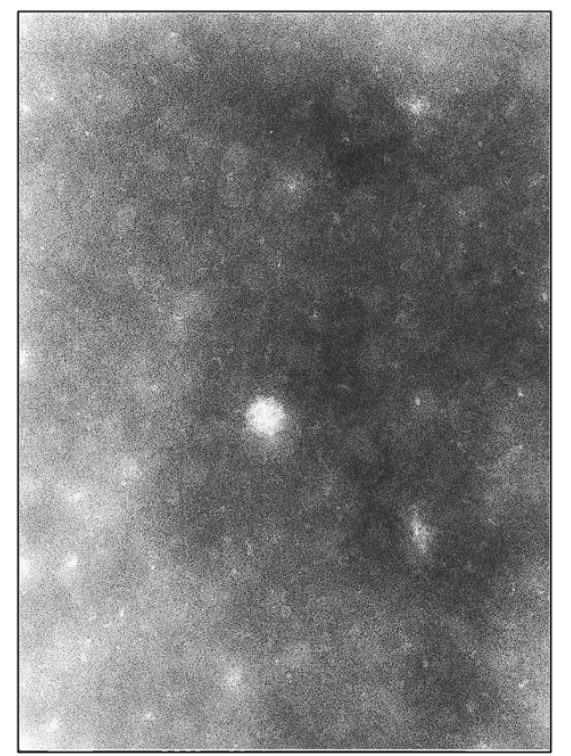

Figure 1. Electron microscopic picture of SK virus. Virus was visualized by staining with $2 \%$ phosphotungstic acid (pH 7.0). Scale bar is $50 \mathrm{~nm}$.
1 GACAGTTACCAGGTAGAGGTGCTCAATGAATCCETCAAGGGCGGIGTCGTGTATTGCYTGGACTACAGTAAGTGGGACTCCACTCAACAC $\begin{array}{llllllllllllllllllllllllllllll} & S & Y & Q & V & E & V & L & N & E & S & L & K & G & G & V & V & Y & C & L & D & Y & S & K & \text { W } & D & S & T & Q & H\end{array}$

91 CCGGCCGTTACAGCTGCATCCTTGGCAATCCTTGAGCGGCTGTCTGAAGCGACACCAATAACAACATCAGCCGTTGAGCTGCTCTCATCA P A $\quad$ V T $T$ A A A S L L A I I L $E$ E $R$ L L 81 CCAGCACGTGGCCACTTAAATGACATAATCTTTGTCACAAAGTCTGGACTCCCTTCGGGCATGCCATTCACTAGTGTTGTCAACTCTCTT P A R G G H L N D I I F V T K S S G L P S G M P F T S V V N S L 271 AACCATATGACATACTTTGCGGCTGCAGTGCTGAAGGCCTATGAACAACATGGGECACCTTACACTGGTAATGTGTTTCAGGTGGAAACT

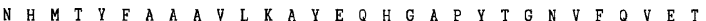
361 GTTCACACATATGGTGATGATTGCATATACTCTTTGTGCCCGGCAACCGCCTCCATCTTTGAAACTGTTCTGGCCAACCTCAGTGCCTTT V H T Y G 51 GGTTTACGGCCCACCGCTGCCGACAAAACAGACAAAATAGCCCCTACCCACACACCAGTCTTTCTAAAACGAACTCTCACCTGCACACCG

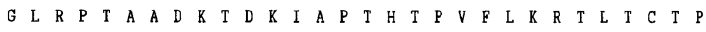
541 AgAGgTATTCGTGGGTTACTCGACATCACGTCAATCAGGCGGCAATTTTTCTGGATCAAGGCCAACCGCACCACAgACATCTCATCOCCG

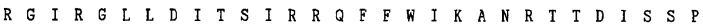
31 CCCGCCTACGACAGGGAGGCCCGGAGTGTGCAGCTTGAAAATGCCCTGGCTTACGCTTCACAACACGGCCACGCAATCTTTGAGGAGATT $\begin{array}{lllllllllllllllllllllllllllllll}P & A & Y & D & R & E & A & R & S & V & Q & L & E & N & A & L & A & Y & A & S & Q & H & G & H & A & I & F & E & E & I\end{array}$

721 GCCGAAATTGCCAAGAGGACGGCACAGTCAGAAGGGTTAGTGCTCACCAATGTGAATTATGACCAGGCTCTCGCCACCTACGAAGCGTGG

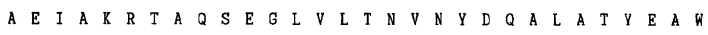

811 TTTATAGGTGGTACAGGCACCGGCCAAGATAGCGCCAGTGAGGAGACTACCAAATTAGTGTTTGAAATGGAGGGCTTGGGCCAGCCACAG F $I$ I $G$ G 901 TCCCAAAGGGACCAACAGGTTATGGAACAGGTTGTCACCCCOCAGGACACCATTGGACCAACGAGTGCACTTTTACTGCCCACTCAAGTT $S$ Q $R$ R D Q Q V GAGACACCAAACGCTAGTGCTCAGCGTGTGGAACTTGCAATGGCCACAGGAGCAGTGACCAGCAATGTGCCCAACTGTATCCGGGAATGT E T $P$ P N A S A Q $R$ R V E L A A M A T G A V T $S$ S N V P N C I R E C TTTGCTGCGGTCACCACAATACCATGGACCACCCGGCAAGCAGCAAATACCTTTCTCGGTGCCATACACCTGGGCCCACGCATCAATCCC $\begin{array}{lllllllllllllllllllllllllllllll} & \text { A } & A & A & V & T & T & I & P & W & T & T & R & Q & A & A & N & T & F & L & G & A & I & H & L & G & P & R & I & N & P\end{array}$ TACACTGCACACCTGAGCGCGATETTTGCTGGTTGGGGGGGGGCTTTCAGGTGCGGGTCACAATCTCAGGTTCCGGGCTCTTTSCTGGT Y T A H L S A M F A G W G G G F Q V V R V T I S G S G L E A G CGAGCCATCACTGCCATCCTECCACCAGGTGTCAACCCAGCTGCTGTTCAGAACCCGGGTGTCTTCCCCCATGCGTTTATTGCTCGCATC $\begin{array}{lllllllllllllllllllllllllllllll}R & A & I & T & A & I & L & P & P & G & V & N & P & A & A & V & Q & N & P & G & V & F & P & H & A & F & I & D & A & R\end{array}$ ACAACTGACCCCATACTGATCAACTTACCTGACATCCGGCCCATTGATTTTCACCGTGTAGATGGGGACGACGCGACAGCGTCTGTGGGG $\begin{array}{llllllllllllllllllllllllllllll}T & T & D & P & I & L & I & N & L & P & D & I & R & P & I & D & F & H & R & V & D & G & D & D & A & T & A & S & V & G\end{array}$ TTATGGGTCGCGCAGCCCTTGATCAACCCGCTTCAAACTGGCTCCGTATCCACATGTTGGCTTAGTTTTGAAACACGGCCTGGCCCTGAC L W V TTTGATTTTTGTCTCCTCAAAGGCCCCCGAGCAGGAAATGGACAATGGGATATCACCTGOCAACCTGTTGCCACGCGCCCTCGGGCGTTC

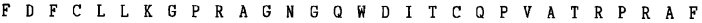
TCGGGGCAACGCCTCGGCGGTCGTGTTGTGGGGCTGETTGTGGTAGCTGCGGCCGAGCAAGTCAACCACCACTTCGGCGCAAACTCCACC $S$ G Q R R L G G R V V G G L V V V A A A A E ACACTTGGTTGGTCAACACTGCCTATTGAGCCAATTGCAGGGGCAGTTTCTTGGTACGATGACAACAATGAACATACCAAGATACGGGGC T L $G$ W $S$ T L $L$ P I E CTTTTGAGTGCACAAGGAAAGGGCATCATCTTCCCAAACATTGTCAACCACTGGACAGATGTGTCATTGTCCGCCAAGACCTCEGGGCAG L L S A Q G K G I I F P N I V N H W T D V S L S A K I S G Q ACAACTATCCCCATAGCTGCGGACAACCTCAACAACAGCCCTGGTGCAGCTGGTCCGGTAGTTATGTTTGAAAACAATGGAGATGTCAAT

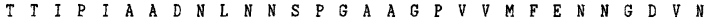
GAGTCCACGGCAAACCATGGCATACTAACCECTGCGTCGCATGACTTCACATCGCTGTCCCAAACTTTCGATGCAGCCGGGTTGTGGGT E S I A N H H $G$ I L $L$ T A A TGGATGCCATGGACCCGGAACAAACCTGACGGGCGTTCAAACACAAATGTCTACATTACTCCAACTTGGATTAATGGCAACCCTGCCCGT

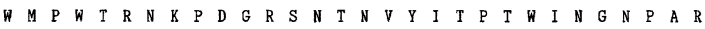
CCCATACATGAGAATGTACCAACATGGTTGGGACTAATTTTCAATTCGGTGGCACTGGGACAAACAACATCATGTTATGGCAGGAGCAA $P$ I H E K C T N M V G T N CACTTCACATCTTTCCCAGGTGCTGCAGAGGTGTACTGTTCACAATTGGAGAGCACAGCAGAAATGTTCCAAAACAATGTGGTCAACATT H F T S S F P P $G$ A A E E V V Y C C S Q L L E S T A A E M F Q N N CCAGCAAACCAAATGGCAGTCTTCAACGTAGAAACTGCAGGTAACACATTCCAGATTGGGATCTTGCCAAACGGCTATTCAGTCACCAAT $\begin{array}{llllllllllllllllllllllllllllll}P & A & N & Q & M & A & V & F & N & V & E & T & A & G & N & T & F & Q & I & G & I & L & P & N & G & Y & S & V & T & N\end{array}$ GCAGCAATTGGCACACATCAACTCCTTGACTACGAGACTAGTTTTAGATTTGTTGGACTTTTCCCCCCAAAGCACTTCACTTCAAGGGCCC $\begin{array}{llllllllllllllllllllllllllllllll}A & \text { A } & I & G & T & H & Q & L & L & D & Y & E & T & S & F & R & F & V & G & L & F & P & Q & S & T & S & L & Q & G & P\end{array}$ AATGGGAACGCTGGACGGGCOGTCAGGTTCTTAGAATAATGTCTTGGTTTACTGGAGCAGCCTTGGCCGCCGGCTCTGTCGTGGACATG N G N A G GCCGGCACAATATCTTCAATTGTTGCACAACACAGACAACTTGATTTGATGGCACAGGCAAACCGCATACAACAGGATTGGGTAAACAGA A $G$ T I S S I V A Q H $R$ CAAGAGGCACTCATCACTCGAGGACAGGACATCTCCCGCGACCTTGCTGTTAATGGAACGGCGCAACGCGTCGACTCACTGGTCAAAGCG Q E GGGTTCACCCCTGTTGATGCTCGCAGATTGGCGGGCGGCACGGAGAGCGTGCAGTACGGCCTGCTGGACAGGCCTATACTGCAACGTTCG $\begin{array}{lllllllllllllllllllllllllllllll}G & F & T & P & V & D & A & R & R & L & A & G & G & T & E & S & V & Q & Y & G & L & L & D & R & P & I & L & Q & R & S\end{array}$ GTGCTGTCGGGCATCTCAGAGACTCGGCACTTGCAGTCTATECAAGGTGCCTTGAGTGCTTTTAAAAATGGATCCAGCTACEGAGCOCCT $V$ L S G I S E T R H L Q S M Q G A L L S A F K K N G S S CCGCCCCAGCGGGCTTCGTTAACCCAAATTATCAGGCTTCACCACCAAAGCTAACACTTGGCCCCAGACCCCCGGTGACTAATGTATAAT P P Q R R A S L L T Q I I I R L H H Q

GATGATTAGGITTTCCCAAGATAATCAAATTTCCACTCTCAGATATCTTTACTTTTCTTTTCTTTTCCCATGGTACCACACGCGTTCGGG

3151 TGGATAATTGTGGTTAAGCGACCACAGCCATGATCCTCTGGAAAGGGAAAAAG

Figure 2. Partial nucleotide and amino acid sequences of SK virus. The nucleotide and its deduced amino acid sequences of the $3^{\prime}$ end with $3.2 \mathrm{~kb}$ was determined. Conserved sequences, GLPSG and YGDD, of RDRP and GSGVFAGR of capsid protein are underlined. Amino acid sequences of capsid protein and ORF2 start with new numbering and the first amino acid of capsid protein was denoted with a bold character. Termination codons of each ORF1 and ORF2 are marked with *. 
fragments were ligated with pMOSBlue T-vector, and transformed to $E$. coli competent cells according to manufacturer's protocol (Amersham Life Science).

\section{Nucleotide sequence accession numbers and ana- lysis}

Sequence data from this study have been deposited to EMBL/GenBank data libraries. The accession numbers for the various viruses are M86379 (FCV), M87611 (NV/ 8FiiA/68/US), L07418 (Southampton/91/UK), L23828 (KY89/Japan), L23831 (SMA/76/US), L23830 (Oth-25/89/ Japan), U22498 (MX/89/Mexico), U73124 (Parkville),
X86559 (Plymouth/92/UK), X86557 (Lordsdale virus), U50825 (Pretoria/MK17/94/SA), U43287 (Vanderbijlpark/ 313616/SA), and X86560 (Manchester virus/93/UK). Nucleotide sequences and amino acid sequences were analyzed by computer program DNA-SISTM and PROSISTM (Hitachi-Amersham Ltd).

\section{Results and Discussion}

\section{Electron microscopic examination}

The HuCV was identified with the characteristic mor-

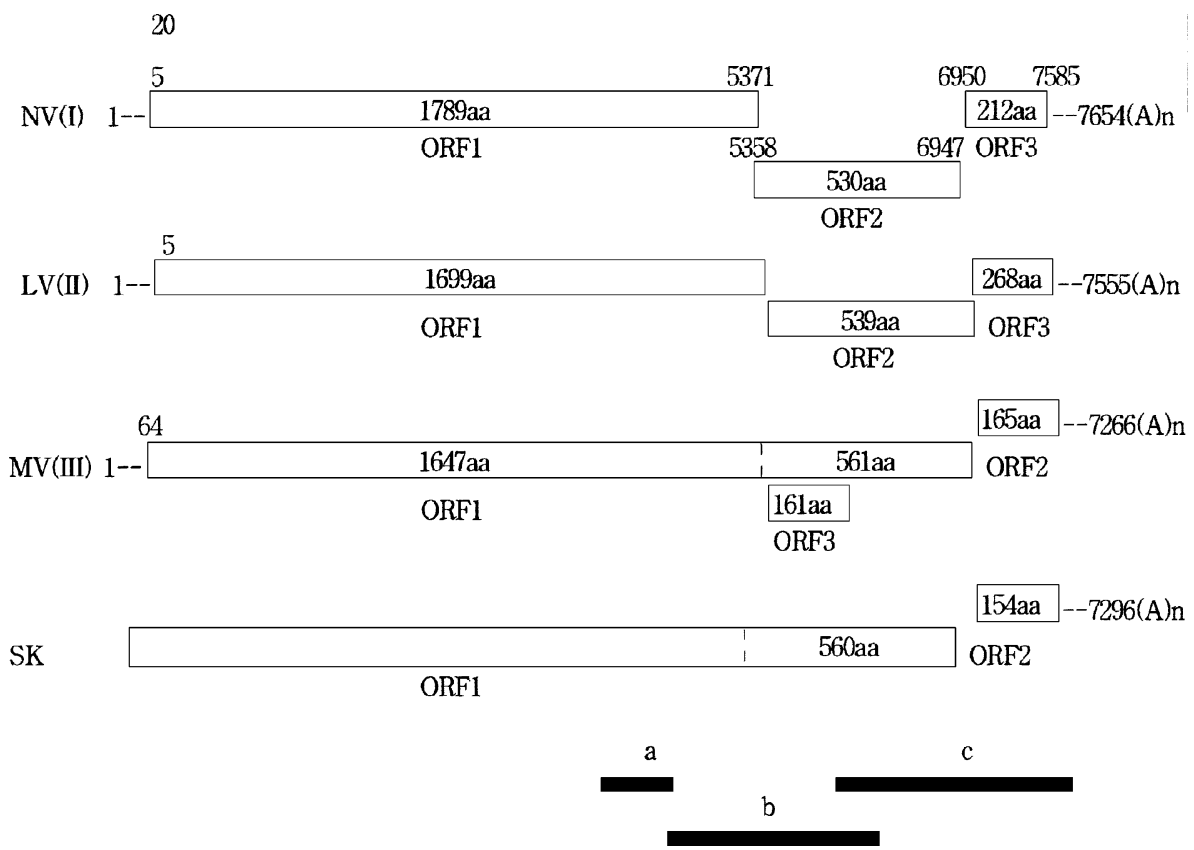

Figure 3. Comparison of the genomic organization of SK virus with HuCVs. Genomic organization of genogroup I (Norwalk virus, NV), II (Lordsdale virus, LV), III (Manchester virus, MV) (EMBL/GenBank data libraries) and SK virus was predicted from the analysis of nucleotide sequence. The bold lines at the bottom of figure indicate the locations of the $3^{\prime}$ partial $c D N A s$ of SK virus obtained from RT-PCR and cDNA libraries $(a, b$, and $c$ denotes cDNA obtaind by each primers of Table 1). All cDNAs were obtained from the stool specimen of a single child with gastroenteritis, and cloned into T-vector and pBlueScript KS.

Table 2. Comparison of percentages of nucleotides and amino acids identity of the RNA-dependent RNA polymerase region of SK virus to other calicivirus

\begin{tabular}{|c|c|c|c|c|c|c|c|c|c|c|c|c|}
\hline & FCV & RHDV & $\mathrm{NV}$ & SHV & SMA & $\mathrm{MX}$ & OTH & $\mathrm{Sa} / 82$ & MV & Vanderbijl & Pretoria & SK \\
\hline FCV & & 54 & 53 & 55 & 55 & 56 & 57 & 59 & 58 & 58 & 57 & 57 \\
\hline RHDV & 40 & & 52 & 54 & 54 & 55 & 56 & 58 & 59 & 57 & 58 & 60 \\
\hline NV (I) & 37 & 35 & & 77 & 62 & 61 & 62 & 55 & 52 & 55 & 54 & 55 \\
\hline SHV (I) & 36 & 36 & 93 & & 65 & 66 & 67 & 56 & 52 & 55 & 54 & 55 \\
\hline SMA (II) & 35 & 35 & 57 & 60 & & 78 & 79 & 55 & 56 & 55 & 55 & 55 \\
\hline MX (II) & 37 & 34 & 57 & 61 & 88 & & 96 & 55 & 56 & 53 & 53 & 55 \\
\hline OTH (II) & 37 & 34 & 58 & 62 & 90 & 96 & & 55 & 56 & 53 & 54 & 56 \\
\hline $\mathrm{Sa} / 82$ (III) & 47 & 38 & 34 & 36 & 36 & 36 & 37 & & 89 & 70 & 68 & 68 \\
\hline Manchester (III) & 48 & 38 & 35 & 36 & 36 & 36 & 37 & 99 & & 69 & 69 & 69 \\
\hline Vanderbijl & 46 & 41 & 34 & 35 & 36 & 36 & 36 & 74 & 73 & & 95 & 85 \\
\hline Pretoria/SA & 46 & 42 & 34 & 36 & 35 & 36 & 36 & 75 & 74 & 97 & & 86 \\
\hline SK & 46 & 42 & 34 & 36 & 35 & 36 & 36 & 75 & 74 & 97 & 99 & \\
\hline
\end{tabular}

The numbers in the upper triangle show the percent identity of the aligned nucleotide sequences, and the number in the lower triangle show the percent identity of the aligned amino acids. Sequences of viruses were obtained EMBL/GenBank Data Libraries. Roman number in paranthesis indicates the type of genogroup. 
phology by electron microscopic examination of stool that was positive for HuCV by RT-PCR. SK (Seoul, Korea) virus showed the typical HuCV appearance with a diameter of around $35 \mathrm{~nm}$ (Figure 1).

\section{Cloning and partial genome organization of SK virus}

Nucleotide sequence of $3.2 \mathrm{~kb}$ fragment of the $3^{\prime}$ end from three overlapping cDNA clones which were obtained by RT-PCR and CDNA library of RNA were determined (Figure 2). Sequence analysis showed that CDNA of the clones spanned from the RNA polymerase region in ORF1 to the 3 ' poly A tail. Figure 3 shows the genomic organization of SK virus compared with other types of HuCVs. The nonstructural and capsid protein coding sequence were fused in a single ORF as observed in Manchester type or RHDV. The ORF2 was located at the $3^{\prime}$ end of genome. The AUG initiation codon of ORF2 is overlapped by one base by the termination codon of ORF1. The size of ORF2 (462 nucleotides, 154 amino acids) is larger than that of RHDV (117 amino acids), but smaller than those of the most HuCVs (165-268 amino acids). The ORF3 shown in Manchester virus was not found in SK virus.

Sequence analysis of SK virus in RDRP region and capsid region (Table 2) compares the nucleotide and predicted amino acid identity of the RDRP region of SK virus and other caliciviruses. SK virus showed amino acid and nucleotide identities of $74-75 \%$ and $68-69 \%$ respectively with those of genogroup III viruses, while showing $34-36 \%$ and $55-56 \%$ identities respectively with those of other human caliciviruses. It should be noted that the SK virus displays higher percentage of sequence similarity with animal viruses (FCV, RHDV) than genogroup I (NV) and genogroup II (SMA, MX, OTH), which is a typical feature of genogroup III. Vanderbijlpark and Pretoria isolates recently found in South Africa but ungrouped due to insufficient data which comprise only about the short region of RDRP showed the highest identities $(97 \%$ and $99 \%$ in amino acid sequences, $85 \%$ and $86 \%$ in nucleotide sequences) with SK virus.

Table 3. Comparison of percentages of amino acid identity of the capsid proteins of SK virus and the other caliciviruses

\begin{tabular}{|c|c|c|c|c|c|c|c|}
\hline \multirow{2}{*}{$\overline{\mathrm{NV}}$} & \multirow[t]{2}{*}{ NV } & \multicolumn{2}{|c|}{ LV FCV } & \multicolumn{4}{|c|}{ RHDV MV Parkville Plymouth SV } \\
\hline & & & & & & & \\
\hline LV & 47 & & & & & & \\
\hline FCV & 23 & 29 & & & & & \\
\hline RHDV & 24 & 33 & 28 & & & & \\
\hline MV & 24 & 32 & 29 & 26 & & & \\
\hline Parkville & 23 & 32 & 27 & 24 & 78 & & \\
\hline Plymouth & 24 & 32 & 29 & 26 & 99 & 78 & \\
\hline SHV & 24 & 32 & 29 & 26 & 99 & 78 & 98 \\
\hline SK & 31 & 32 & 29 & 32 & 44 & 46 & 44 \\
\hline
\end{tabular}

The numbers represent the percent identity of the aligned amino acids of capsid region
Table 3 shows an overall sequence identity of the capsid protein of SK virus and those of animal and human calicivirus. The capsid protein of SK virus (560 amino acids) was longer than SRSVs (530-548 amino acids) but shorter than Manchester type (561 amino acids) (Numata et al., 1997). Amino acid identities between SK virus and other caliciviruses range $29-46 \%$. Although the sequence identities between SK and Manchester strains (genogroup III) are higher than other caliciviruses, the identities are only $44-46 \%$, which is quite low to be placed in the same genogroup. Figure 4 shows a comparison of direct amino acid between the capsid proteins of SK virus and genogroup III. N-terminus region (Region $1,54-59 \%$ ) and C-terminus region (Region 3, 50-52\%) are more conserved than the central region (Region 2, $15-18 \%)$. There is a conserved motif of GSGVFAGR in

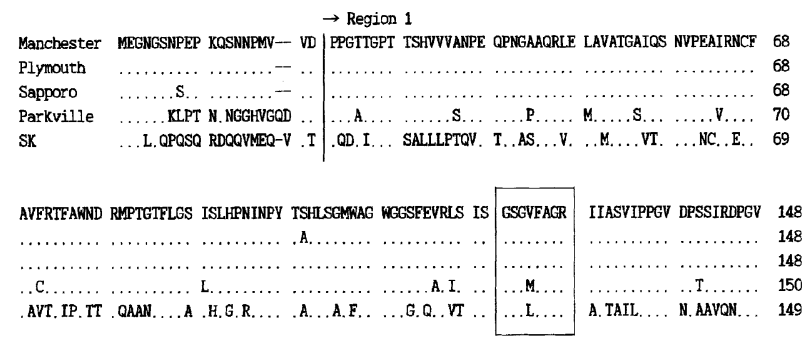

LPHAFVDARI TEPVSFMTPD VRAVDYHPRD GAEPTCSLGF WVYOPLLNPF STTAVSTCWV SVETKPGGDF DFCLLRPPGQ 228

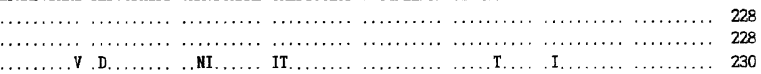

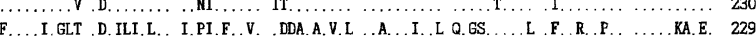

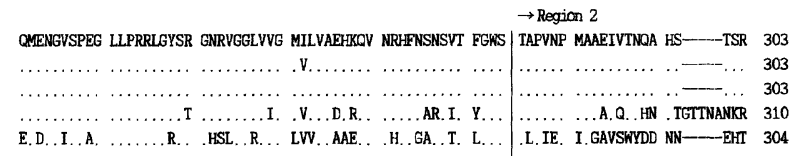

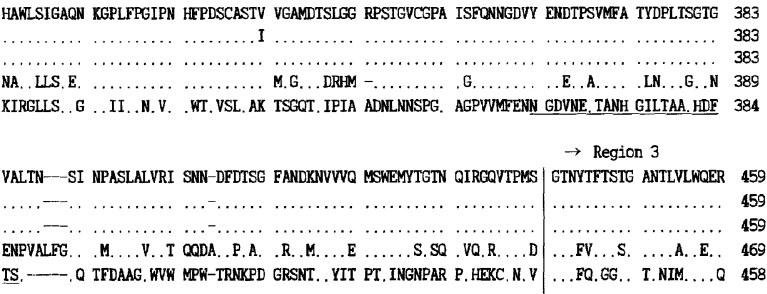
MLSYDGHQAI LYSSOLERTA EYFQNDIVNI PENSMAVENV ETNSASFQIG IRPDGYMVTG GSIGWNVLE PETRFQYVGI 539

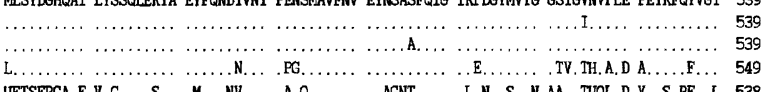

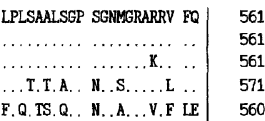

Figure 4. Alignment of the amino acid sequences of capsid regions of SK virus and other strains included in HuCV genogroup III. Identical amino acid residues shown as dots and dashes are used to adjust alignment. The open box indicates conserved regions among HuCV genogroup III and animal caliciviruses. The underlined region indicates an additional amino acid insertion site in the variable region. 
$\mathrm{N}$-terminus region. However, $\mathrm{V}$ in this region was changed to $L$ and $M$, in SK and Parkville strain. As in Manchester virus, capsid protein of SK virus was longer by 30 amino acids than that of other SRSVs, and these additional amino acids are present in central variable region.

Compared to RDRP region which showed a relatively high amino acid identity (74\%) to Manchester virus, deduced amino acid sequence of capsid region of SK virus showed only $44 \%$ identity to that of Manchester virus. In addition, ORF3 appeared in Manchester virus was not found in SK virus. In order to place virus strains into the same genogroup, amino acid sequence identity of $>82 \%$ in the RNA polymerase region, and of $>65 \%$ in capsid region is generally required (Lew et al., 1994; Estes et al., 1997). Vanderbijlpark and Pretoria isolates recently found in South Africa was classified as Manchester-like type in EMBL/Genbank data library. Present investigation revealed that Vanderbijpark and Pretoria isolates showed very high identities (>97\% in amino acids and $>85 \%$ in nucleotides) with SK virus. These may suggest that SK virus together with Vanderbijpark and Pretoria isolates could be a new type different from Manchester type.

\section{Acknowledgements}

This work was supported by the academic research grant of Yonsei University, Wonju College of Medicine for 1998.

\section{References}

Adler, J. L. and Zickl, R. (1969) Winter vomiting disease. J. Infect. Dis. 119: 668-673

Ando, T., Mulders, M. N., Lewis, D. C., Estes, M. K., Monroe, S. S. and Glass, R. I. (1994) Comparison of the polymerase region of small round structured virus strains previously classified in three antigenic types by solid-phase immune electron microscopy. Arch. Virol 135: 217-226

Appleton, H. (1987) Small round viruses: Classification and role in food-borne infections. In Novel Diarrhoea Viruses. (Back, G., Whelan, J., eds) pp 238-249, Wiley, Chichester

Carter, M. J., Milton, I. D., Meanger, J., Bennett, M., Gaskell, R. M. and Turner, P. C. (1992) The complete nucleotide sequence of a feline calicivirus. Virlolgy 190: 443-448

Cubitt, W. D., Blacklow, N. R., Herrmann, J. E., Nowak, N. A., Nakada, S. and Chiba, S. (1987) Antigenic relationship aming human calicivirus and Norwalk virus. J. Infect. Dis. 156: 806814

Estes, M. K., Atmmar, R. L. and Hardy, M. E. (1997) Norwalk and related diarrhea viruses. In Clinical virology. (Richman, D. D., Whitley, R. J., Hayden, F. G. eds). pp 1073-1095, Churchill Livingstone, New York.

Jiang, X., Graham, D. Y., Wang, K. and Estes, M. K. (1990) Norwalk virus genome cloning and characterization. Science
250: $1580-1583$

Jiang, X., Wang, M., Graham, D. Y. and Estes, M. K. (1992a) Expression, self-assembly, and antigenicity of the Norwalk virus capsid protein. J. Virol. 66: 6527-6532

Jiang, X., Wang, M., Graham, D. Y. and Estes, M. K. (1992b) Detection of Norwalk virus in stool by polymerase chain reaction. J. Clin. Microbiol. 30: 2529-2534

Jiang, X., Wang, M., Wang, K. and Estes, M. K. (1993) Sequence and genomic organization of Norwalk virus. Virology 195: 51-61

Kapikian, A. Z., Wyatt, R. G., Dolin, R., Thornhill, T. S., Kalica, A. R. and Chanock, R. M. (1972) Visualization by immune electron microscopy of a $27 \mathrm{~nm}$ particle associated with acute infectious nonbacterial gastroenteritis. J. Virol. 10: 1075-1081

Lambden, P. R. and Clark, I. N. (1995) Genome organization in the Calciviridae. Trends Microbiol. 3: 261-265

Levett, N. P., Gu, M., Luan, B., Fearon, M., Stubberfield, J., Jamieson, F. and Petric, M. (1996) Longitudinal study of molecular epidemiology of small round_structured viruses in a pediatric population. J. Clin. Microbio. 34: 1497-1501

Lew, J. F., Petric, M., Kapikian, A. Z., Jiang, X., Estes, M. K. and Green, K. T. (1994) Identification of Minireovirus as a Norwalk_like virus in pediatric patients with gastroenteritis. $J$. Virol. 68: 3391-3396

Madeley, C. R. and Cosgrove, B. P. (1976) Caliciviruses in man. Lancet 1: 199

Matson, D. O., Zhong, W. M., Nakata, S., Numata, K., Jiang, X., Pickering, L. K., Chiba, S. and Estes, M. K. (1995) Molecular characterization of a human calicivirus with sequence relationships closer to animal caliciviruses than other known human caliciviruses. J. Med Virol. 45: 215-222

Matsui, S. M., Kim, J. P., Greenberg, H. B., Wanchuang, S., Quming, S., Johnson, P. C., DuPont, H. L., Oshiro, L. S. and Reyes, G. R. (1991) The isolation and characterization of a Norwalk virus-specific cDNA. J. Clin. Investigation 87: 14581461

Meyers, G., Wirblich, C. and Thiel, H. J. (1991) Rabbit hemorrhagic disease virus. Molecular cloning and nucleotide sequencing of a calicivirus genome. Virology 184: 664-676

Middleton, P. J., SzymanSKi, M. T. and Pietric, M. (1977) Viruses associated with acute gastroenteritis in young children. Am. J. Dis. Child. 131: 733-737

Neill, J. D., Reardon, I. M. and Heinrikson, R. L. (1991) Nucleotide sequence and expression of the capsid protein gene of feline calicivirus. J. Virol. 65: 5440-5447.

Numata, K., Hardy, M. E., Nakata, S., Chiba, S. and Estes, M. K. (1997) Molecular characterization of morphologically typical human calicivirus Sapporo. Arch Virol. 142: 1537-1552

Sanger, F., Nicklen, S. and Coulson, A. R. (1977) DNA sequencing with chain_terminating inhibitor. Proc. Natl. Acad. Sci. USA. 74: 5463-5467

Schaffer, F. L., Bachrach, H. L., Brown, F., Gillesple, J. H., Burroughs, J. N., Madin, S. R., Povey, C., Scott, F., Smith, A. W. and Studdert, M. J. (1980) Intervirology 14: 1-6 